\title{
La enseñanza de lenguas extranjeras: historia, teoría y práctica
}

\author{
Gabriela Cerdas Ramírez \\ Escuela de Literatura y Ciencias del Lenguaje \\ Universidad Nacional, Costa Rica \\ Jimmy Ramírez Acosta \\ Escuela de Literatura y Ciencias del Lenguaje \\ Universidad Nacional, Costa Rica
}

\begin{abstract}
Resumen
El presente artículo describe y analiza varias teorías de aprendizaje de lenguas extranjeras con el objetivo de guiar al lector a la puesta en práctica de estrategias que le permitan mejorar el proceso de enseñanza/ aprendizaje. En ocasiones, los problemas que se presentan en el proceso de aprendizaje de lenguas extranjeras están relacionados justamente con la imposibilidad que enfrentan algunos profesionales de llevar los enunciados teóricos a la práctica en forma exitosa. Se realiza una investigación con el objetivo de determinar si un grupo de docentes de secundaria conoce y aplica estos principios de tal forma que les permita a los estudiantes enfrentar el proceso de enseñanza-aprendizaje de una manera satisfactoria
\end{abstract}

Palabras claves: lenguas extranjeras, adquisición, aprendizaje, estilos de aprendizaje, estrategias de aprendizaje, modelos pedagógicos

\begin{abstract}
This article describes and analyzes different foreign language learning theories that are aimed to help the reader improve the language teaching and learning process. Sometimes, the problems that arise in the process of learning foreign languages have to do with the impossibility faced by some professionals to bring theoretical concepts into a successful practice. An investigation has been carried out with the objective of determining if a group of high school teachers know and apply these theoretical principles so that students can appropriately cope with the learning process.
\end{abstract}

Key words: foreign languages, acquisition, learning, learning styles, learning strategies, approaches 


\section{Introducción}

$\mathrm{L}$ a creciente demanda de formar profesionales capaces de comunicarse en un segundo idioma ha puesto en evidencia la necesidad de que las escuelas y los colegios públicos mejoren la calidad de instrucción que se ofrece a los estudiantes en este idioma. Esta necesidad de formar ciudadanos bilingües nace de la realidad que afrontan todos los países en vías de desarrollo que desean incorporarse exitosamente en un mercado globalizado.

Muchos estudiantes tienen su primer contacto con un segundo idioma hasta llegar a las aulas de secundaria - III y IV ciclos-. Es por eso que actualmente los profesores de lengua extranjera de los colegios públicos tienen un papel preponderante en el desarrollo de las habilidades lingüísticas de sus estudiantes, en concordancia con los parámetros establecidos por el Ministerio de Educación Pública (MEP). Cabe destacar que muchos de estos docentes van más allá de lo que establece el currículo de inglés del MEP al tratar de impartir lecciones donde las actividades y ejercicios giran alrededor de un propósito comunicativo (oral o escrito), con el afán de que los estudiantes desarrollen la capacidad de comunicarse efectivamente en un segundo idioma. Sin embargo, esta tarea de lograr que los estudiantes desarrollen buenas competencias lingüísticas es casi imposible si las estrategias y la mediación pedagógica no guardan estricta relación con las teorías más avanzadas sobre el aprendizaje de lenguas.

En este contexto, es necesario que los docentes no solo tengan claras las teorías de aprendizaje de una segunda lengua, sino también que estén en la capacidad de aplicar los principios elementales que les permitan a los estudiantes el aprendizaje del idioma basado en un método más comunicativo y adaptado a sus necesidades. Es por eso que este artículo tiene como objetivo principal determinar si un grupo de docentes de secundaria conoce y aplica principios que les permita a los estudiantes enfrentar el proceso de enseñanza-aprendizaje de una manera satisfactoria.

A continuación se realiza un recorrido por algunas teorías fundamentales para mejorar el proceso de enseñanza-aprendizaje de una lengua extranjera.

\section{Teorías en adquisición de lenguas extranjeras}

Quizás una de las más importantes teorías sobre la adquisición de lenguas extrajeras es el modelo de adquisición del lenguaje propuesto por Krashen y Terrel. Este modelo se divide en cinco hipótesis: 1) adquisición-aprendizaje, 2) orden natural, 3) automonitorización, 4) "Input", y 5) filtro afectivo. Debido a que la hipótesis del orden natural se centra más en adquisición de segundas lenguas, y no tanto en la adquisición de lenguas extranjeras, no se discutirá en este marco referencial. 


\subsection{La hipótesis del aprendizaje-adquisición}

De acuerdo con esta hipótesis, las personas tienen dos maneras distintas para desarrollar la competencia lingüística ya sea a través del aprendizaje o de la adquisición. La adquisición del lenguaje es un proceso similar a la forma en que hemos aprendido nuestra lengua materna. Desde que éramos niños, hemos ido adquiriendo nuestra lengua sin entrenamiento formal. Nadie nos dijo acerca de las reglas gramaticales o de patrones de pronunciación para que pudiéramos aprender a hablar español. En otras palabras, la "Adquisición del Lenguaje es la forma natural de desarrollar la capacidad lingüística, y es un proceso subconsciente" (Krashen y Terrel, 1983, p.2). Así que la adquisición del lenguaje tiene que ver con el aprendizaje inconsciente, en el que se adquiere el lenguaje de forma natural en contextos comunicativos, y no hay ninguna enseñanza formal.

Por otro lado, "El aprendizaje de un idioma se refiere al conocimiento explícito de las reglas, siendo consciente de ello y teniendo la capacidad de hablar de ello" (Krashen y Terrel, 1983, p.2). Se trata de un proceso consciente donde hay instrucción formal, y también hay conocimiento formal de la lengua. Un caso muy concreto de aprendizaje sería la situación a la que se enfrenta un profesor de lengua no nativo, quein ha tenido que tomar diferentes cursos formales en los que ha aprendido una gran cantidad de información relacionada con los diferentes aspectos del idioma: fonología, fonética, morfología, sintaxis, etc.

\subsection{La hipótesis de automonitorización}

Puesto que todas estas hipótesis están relacionadas, hay que tener en cuenta lo que hemos mencionado sobre la hipótesis de la adquisición-aprendizaje. De acuerdo con el modelo monitor, cuando un enunciado es procesado por nuestro sistema de adquisición del lenguaje, el conocimiento que obtenemos del aprendizaje del idioma (aprendizaje consciente) será el encargado de la corrección de nuestras expresiones. Esto no quiere decir que el sistema de adquisición no va a corregir expresiones, porque hay momentos en los que corregimos expresiones en las que utilizamos el sistema de adquisición. Esto significa que a veces usamos lo que Krashen llama la "sensación de corrección" para editar enunciados producidos en nuestra lengua materna y nuestra lengua meta. Según Krashen, el aprendizaje consciente tiene sólo la función de corrección de los enunciados y no la función para iniciar la producción en un segundo idioma (Gass y Selinker, 1994, p.149).

El conocimiento de la lengua que tenemos entra en juego cuando corregimos nuestras declaraciones, ya sea antes, en el momento o inmediatamente después de que hayan sido pronunciadas. Según Hockett (1967), se utilizan dos procesos para corregir nuestras expresiones: corrección externa y corrección interna. La corrección externa es el proceso que tiene lugar cuando se reparan o editan nuestras expresiones después de que se han producido. La edición interna, por el contrario, es un proceso mediante el cual las declaraciones se han corregido o 
editado antes de que se produzcan realmente. Este proceso es de alguna manera menos evidente porque lo que escuchamos es la expresión correcta.

Hay algunos requisitos que deben cumplirse para que esta hipótesis funcione:

1. Tiempo: el orador debe tener tiempo para pensar acerca de las reglas y otros aspectos relacionados con el idioma. Si estamos hablando y se nos da tiempo para pensar en la forma correcta de decir las cosas, estaremos menos propensos a cometer errores.

2. Concentración en la forma: a veces nos centramos más en lo que estamos diciendo y no en cómo lo estamos diciendo. Pero para que la automonitorización funcione, tenemos que centrar nuestra atención no solo en el mensaje sino también en la forma.

3. Conocimiento: si no se tiene conocimiento lingüístico y sociolingüístico de las convenciones que utilizamos para controlar un área en particular, no será probable que podamos corregir algo que está mal (Krashen y Terrel, 1983, p.30).

\subsection{La hipótesis de entrada (Input)}

Esta hipótesis afirma que las personas pueden realmente adquirir el lenguaje cuando entienden y están expuestas a un estímulo lingüístico que se encuentra un poco más allá de su nivel actual de competencia, lo que se llama (i + 1) donde i es el nivel de estímulo lingüístico y " 1 " tiene que ver con un estímulo lingüístico que forma parte de la siguiente etapa de aprendizaje del individuo.

El estímulo lingüístico que se da al estudiante debe tener estructuras conocidas por este y debe incluir también algunas estructuras o vocabulario que forman parte de la siguiente etapa de aprendizaje. Esto significa que para que los estudiantes mejoren su nivel de competencia, los profesores tienen que proporcionarles material lingüístico más allá de su nivel de competencia.

\subsection{La hipótesis del filtro afectivo}

Quizás uno de los principios más importante en la enseñanza de lenguas es la hipótesis del filtro afectivo (Krashen y Terrel, 1983, p.1) de acuerdo con la cual cuando un alumno tiene una visión positiva hacia el proceso de aprendizaje, tendrá más éxito en la adquisición de la lengua extranjera que las personas que no están motivadas. Esto significa que cuando el filtro afectivo está bajo, los estudiantes están más abiertos a aprender y adquirir la lengua con éxito. Es decir, es probable que los estudiantes busquen situaciones en las que pueden obtener más conocimiento y poner en práctica lo que han aprendido con confianza con hablantes de la lengua meta. Además, las personas con un filtro afectivo bajo son más receptivas a la información que reciben (Krashen y Terrel, 1983, p.39). Por otro lado, las personas con un filtro afectivo alto tienden a tener problemas con la adquisición de lenguas porque están estresadas haciendo muchas cosas a la vez, no suelen estar interesadas 
en la búsqueda de situaciones en las que pueden practicar lo que aprenden debido a su falta de motivación, al estrés o a la falta de tiempo. Es importante, por lo tanto, propiciar un ambiente de clase agradable y en la medida de las posibilidades libre de estrés, el cual permitirá que los estudiantes mantengan un filtro afectivo más bajo y por ende aumenten su capacidad receptora y productiva.

\section{Estilos de aprendizaje y estrategias de aprendizaje}

Hay otros dos componentes que se deben destacar en la adquisición de lenguas: los estilos de aprendizaje y las estrategias de aprendizaje. En concreto, estamos hablando de la forma cómo los individuos aprenden o adquieren un idioma. La principal razón para la inclusión de estas teorías en la enseñanza de lenguas en las aulas se basa en la suposición de que los estudiantes tomen conciencia de los diversos aspectos de la adquisición del lenguaje que serán capaces de transferir a otras situaciones de aprendizaje de idiomas, facilitando así su propio proceso de aprendizaje.

Los estudiantes serán capaces de disfrutar de la experiencia de aprender un idioma y de tener éxito en el proceso de aprendizaje si son conscientes del proceso de adquisición del lenguaje, sus estilos de aprendizaje y sus estrategias de aprendizaje. Otras razones para la inclusión de estas teorías en nuestros salones de clase son:

1. Los estudiantes comprenderán cómo los seres humanos se comunican y cómo el lenguaje se adquiere y se aprende.

2. Los estudiantes sabrán que hay estrategias para facilitar su proceso de aprendizaje.

3. Los estudiantes aprenderán que la lengua meta se puede utilizar para adquirir nuevos conceptos e información en varias áreas.

Sabemos que todos somos diferentes y por eso no procesamos y percibimos la información de la misma manera. Todos, por lo tanto, podemos tener diferentes estilos de aprendizaje. Brown (1994) propone la siguiente definición: "Los estilos de aprendizaje son las características generales de funcionamiento intelectual y de personalidad que se refieren a usted como individuo y que le permiten diferenciarse de otra persona" (p. 104).

Según Brown (1994), los educadores y psicólogos han llegado a una larga lista de estilos de aprendizaje; sin embargo, pocos de estos estilos han recibido la atención de los investigadores de una segunda lengua en los últimos años. Brown identifica siete diferentes estilos de aprendizaje (p. 105):

\subsection{Campo-dependiente}

La personas campo-dependientes tienden a confiar en los marcos externos de referencia en el procesamiento de la información. También perciben un campo 
como un todo y tienen grandes habilidades interpersonales en las relaciones sociales (Ellis, 1994, p.114).

Según Brown (1994), las personas que son campo-dependientes tienden a ser más sociables, tienden a derivar su propia identidad de las personas que les rodean, y suelen ser más enfáticas y perceptivas de los sentimientos y los pensamientos de los demás (p.106).

\subsection{Campo-independiente}

Las personas campo-independientes se basan en los marcos internos de referencia en el procesamiento de la información. Por lo general, son más analíticas lo que significa que ellas perciben un campo en términos de sus componentes. Son más independientes que las personas campo-dependientes y no son tan socialmente conscientes (Ellis, 1994, p.114). Según Brown (1994), las personas con estas características tienden a ser más independientes, competitivas y seguras de sí mismas (p.106).

\subsection{Reflexivo}

Se refiere básicamente a las personas muy cuidadosas con todos los detalles antes de que realmente puedan decidir sobre una solución (Brown, 1994, p.112). Esto significa que estas personas se concentran mucho en hacer las cosas bien, que se sienten inseguras y prestan minuciosa atención a los detalles.

\subsection{Impulsivo}

Se refiere a personas que no toman mucho tiempo para pensar en la solución o la respuesta que van a dar. Cuando nos enfrentamos a este tipo de alumnos, los profesores deben advertirles que, además de tiempo, la precisión lingüística es también importante y que deben pensar las cosas antes de expresar sus ideas. A veces tenemos alumnos que nos dan una respuesta correcta segundos después de haber formulado una pregunta, mientras que en otros casos hay estudiantes que no proporcionan una respuesta precisa. Estos tipos de estudiantes deben ser alentados a tomar un poco más de tiempo antes de que den su respuesta u opinión.

\subsection{Visual}

A las personas que son visuales les resulta fácil aprender mediante la lectura y el estudio de gráficos, dibujos y otro tipo de información gráfica (Brown, 1994, p.113). Este tipo de personas solo pueden aprender haciendo organizadores gráficos y/o escribiendo la información. 


\subsection{Auditivo}

Este estilo se refiere a las personas que se caracterizan por una preferencia para escuchar conferencias, cintas de audio y a otras personas (Brown, 1994, p.113). Hay estudiantes que pueden memorizar, interiorizar y aprender la información con sólo escuchar a sus maestros, ya que no les es necesario escribir ningún tipo de nota. La información que se les da se procesa inmediatamente y no necesitan transferir esta información al papel.

\section{Cultura}

Uno de los aspectos que cobra mayor importancia en el desarrollo de un método para la enseñanza de cualquier idioma es la incorporación del aspecto cultural. Dado que todos satisfacemos nuestras necesidades físicas y psicológicas de diferente manera, tenemos que entender cómo la gente de la cultura meta satisface las suyas, para de esta forma comprender sus valores, creencias y prioridades. De esta manera, sabríamos cómo comportarnos lingüística y socialmente en cualquier circunstancia o situación que corresponda a las demandas sociales de la cultura meta.

Uno de los aspectos más importantes en el proceso de enseñanza y aprendizaje de una segunda lengua o una lengua extranjera es la cultura porque nos permite entender los diferentes procesos sociales, comportamientos y puntos de vista de los individuos de la cultura meta. De esta manera, desarrollamos habilidades que se necesitan para comunicarse efectivamente con los hablantes de esa lengua. Como Seelye establece:

Cuando la comunicación se realiza entre personas con diferentes visiones del mundo, se requieren habilidades especiales, si se pretende que los mensajes recibidos tengan concordancia con los mensajes enviados. La habilidad principal más importante es entender el contexto en el que la comunicación se lleva a cabo. Este contexto es en gran medida determinado por la cultura. $(1993$, p.1)

Seelye ha establecido que existen diferentes definiciones sobre lo que es cultura, y que estas dependen en gran medida del campo de estudio. Para efectos de la enseñanza, el autor propone un concepto amplio que se ocupa de todos los posibles campos de estudio que la cultura puede abordar. Seelye afirma que la mayoría de los profesionales están dedicados al concepto de cultura que se ocupa de las bellas artes, la geografía, la historia, los deportes, etc.; sin embargo, afirma que estos aspectos no permitirán a los estudiantes ni entender la lengua meta como fenómeno cultural complejo, ni interactuar apropiadamente (1993, p.14).

Jone Donne, citado en Brown (1994, p.163) propone la siguiente definición de cultura: "La cultura es una forma de vida. La cultura es el contexto en el que existimos, pensamos, sentimos, y nos relacionamos con los demás. Es el "pegamento" 
que une a un grupo de gente". Tenemos que entender que las personas viven de diferentes maneras, y que la forma en que vivimos en Costa Rica definitivamente no es la misma que en otros países por diferentes razones. La cultura es lo que mantiene una nación o un país unido por medio de la música, la vestimenta, las tradiciones, las comidas, etc.

La forma en que actuamos es distinta de la forma en que las personas actúan en una sociedad diferente. Esto depende sobre todo de los aspectos culturales, tales como historia, cosmovisión, valores geográficos, avances tecnológicos y crisis contemporáneas. Estos aspectos afectan la manera en que somos, y es por eso que es importante para los estudiantes de segundas lenguas o lenguas extranjeras identificar, entender y aceptar estas diferencias de comportamiento.

La importancia de la enseñanza de la cultura tiene que ver con el hecho de que la cultura y el lenguaje están estrechamente entrelazados, por lo tanto no se puede separar uno del otro sin perder significado.

\section{Enfoques de enseñanza en lenguas extranjeras}

Al decidir sobre un determinado enfoque o método, es muy importante saber que no existe un método perfecto para enseñar una clase. Cada maestro debe desarrollar las habilidades necesarias para combinar diferentes enfoques con el fin de satisfacer las necesidades de la clase o grupo al que se pretende enseñar. Los maestros que escogen y eligen diferentes aspectos de los distintos métodos se puede decir que tienen una filosofía de enseñanza ecléctica. La importancia de hacerlo es que el maestro, de alguna manera, selecciona las técnicas más adecuadas para la enseñanza de un grupo específico al tratar de cumplir con todos los diferentes estilos de aprendizaje mientras enseña. A continuación se realizará una pequeña descripción de los métodos más tradicionales para la enseñanza de lenguas extrajeras:

\subsection{El Método de Traducción Gramatical}

Este método se empezó a utilizar desde el año 1920 y se centró en la traducción. Los maestros que utilizaban el Método de Traducción Gramatical creían que el propósito fundamental de aprender un idioma extranjero era desarrollar las habilidades de lectura y escritura en la lengua meta. Para alcanzar este propósito los alumnos tenían que aprender las reglas de gramática y vocabulario de esta lengua (Larsen-Freeman, 2000, p.17).

En este enfoque, las instrucciones se dan en la lengua materna de los estudiantes y hay poco uso de la lengua meta. Se les pide que traduzcan las lecturas de la lengua meta a su lengua materna, y también tienen que estudiar la gramática deductiva, lo que significa que el instructor enseña a los estudiantes las reglas de la gramática y los estudiantes tienen que memorizarlas. 
Puesto que el propósito de este método es desarrollar la capacidad de escritura y lectura en la lengua meta, los estudiantes no son capaces de usar el lenguaje para la comunicación (Richards y Rodgers, 1986, p.3), debido a que las técnicas utilizadas se centran principalmente en: traducir pasajes, responder preguntas de comprensión de lectura, utilizar sinónimos y antónimos, reconocer cognados, memorizar y escribir composiciones.

\subsection{Enfoque Directo}

Dado que el Método de Traducción Gramatical no fue efectivo en la preparación de los estudiantes para comunicarse en la lengua meta, el Método Directo se hizo popular. Como Celce-Murcia dijo: "Este [Método Directo] es una reacción al enfoque de la gramática y la traducción y su falla en crear alumnos que pudieran utilizar la lengua extranjera que habían estado estudiando" (1991, p.6).

Los maestros que utilizan el Método Directo quieren que sus estudiantes se comuniquen en esta lengua y con este fin deben aprender a pensar en la lengua meta. Cuando se les enseña con este método, no se les permite utilizar su lengua materna; además, cada vez que una nueva palabra se introduce el maestro demuestra su significado con objetos reales, imágenes o el lenguaje corporal (nunca se utiliza la traducción).

En este método, los estudiantes hablan la lengua meta y se comunican como si estuvieran en situaciones reales; de hecho el programa que se utiliza con el Método Directo se basa en situaciones. La gramática y la cultura se les enseña inductivamente; esto significa que a los estudiantes se les presentan ejemplos y tienen que averiguar la regla. Aprenden vocabulario nuevo, pero la diferencia con el Método de Traducción Gramatical es que los estudiantes usan las nuevas palabras en sus discursos.

\subsection{El Método Audiolingüístico}

El método audiolingüístico, así como el Método Directo, tienen algo en común: el énfasis en producción oral. El Método Directo apunta hacia la adquisición de vocabulario a través de la exposición a situaciones reales, mientras el Método Audiolingüístico enfatiza la adquisición de estructuras gramaticales (Larsen-Freeman, 2000, p.35).

Los maestros que implementan este método creen que los estudiantes tienen que aprender sobre la lengua meta, que les permita usarla de forma automática, sin detenerse a pensar. El vocabulario y la gramática se presentan por medio de diálogos, los cuales se aprenden mediante la imitación y la repetición coral, y el maestro actúa como un director de orquesta dirigiendo y controlando el comportamiento de los estudiantes. El Método Audiolingüístico implica tres conceptos psicológicos que analizaremos brevemente: estímulo, respuesta y refuerzo (Richards y Rodgers, 1986, p.50). Estímulo se refiere a lo que se enseña o se presenta de una lengua extranjera, la respuesta es la reacción de los alumnos 
a los estímulos, y el refuerzo es un elemento vital en el proceso de aprendizaje, ya que dice a los estudiantes si la respuesta es o no adecuada.

\subsection{El Enfoque Cognitivo}

De acuerdo con Celce-Murcia, este enfoque es una reacción a las características conductuales del Método Audiolingüístico (1991, p.7). En este tipo de enfoque de aprendizaje, el lenguaje es visto como la adquisición de la regla y no la formación de hábitos como en el enfoque audiolingüístico. La gramática se puede enseñar deductiva o inductivamente, lo que significa que a veces los estudiantes van a ser informados sobre las reglas gramaticales y en algunos otros casos se les va a pedir que deduzcan las reglas. Las habilidades de lectura y la escritura son tan importantes como escuchar y hablar. Otro aspecto relevante es que a la pronunciación se le resta importancia, porque la perfección es vista como poco realista.

\subsection{Enseñanza Comunicativa de la Lengua}

Los orígenes de la Enseñanza Comunicativa de la Lengua se remontan al Reino Unido a finales de 1960. En ese momento, el enfoque situacional en el que el idioma se enseña mediante la práctica de las estructuras básicas en actividades significativas, fue considerado como el mayor acercamiento a enseñar inglés (Richards y Rodgers, 1986, p.64). Sin embargo, los lingüistas se dieron cuenta de que el enfoque situacional había perdido fuerza, porque tenía la intención de que los estudiantes predijeran el idioma basado en eventos situacionales. Se dieron cuenta de que tenían que estudiar más la lengua y entender cómo los enunciados producidos por un alumno expresaban el significado y la intención del hablante.

Un aspecto muy importante en la enseñanza comunicativa de la lengua es que se centra no sólo en las funciones (las intenciones de la comunicación para expresar ideas, actitudes, etc., situaciones sociales particulares), sino también en las estructuras. A los estudiantes que están aprendiendo con el método de la Enseñanza Comunicativa de la Lengua se les introduce por primera vez a las funciones con el fin de que puedan practicar las distintas estructuras y expresiones. Las funciones que se llevarán a cabo en situaciones de comunicación, la negociación de significado en cooperación, la formación de hipótesis, la puesta en práctica y la utilización de la estrategia de ensayo y error son algunas de las estrategias comúnmente usadas.

En la Enseñanza Comunicativa de la Lengua, el "lenguaje auténtico" (el lenguaje como se usa en contextos reales) debe ser utilizado. Esto significa que los alumnos están expuestos a cualquier tipo de material en el que se presenta el lenguaje auténtico. Los estudiantes deben ver vídeos, leer periódicos y revistas, y navegar por Internet en busca de una práctica adicional fuera de la clase con hablantes nativos, entre otros aspectos. Además, la lengua meta se utiliza no sólo como objeto de estudio sino también como el vehículo para la comunicación en el aula. 
Una parte esencial de este método son los juegos, ya que permiten que los estudiantes apliquen lo que han aprendido para obtener una respuesta inmediata $\mathrm{y}$, por supuesto, divertirse al bajar su filtro afectivo. Los errores son tolerados y vistos como el resultado natural del desarrollo de la comunicación. Los estudiantes usan el lenguaje en actividades comunicativas como juegos, conversaciones, discusiones, debates, obras de teatro y tareas de resolución de problemas. Este tipo de actividades debe cumplir con tres funciones de acuerdo con Johnson y Morrow (1981): falta de información, elección y retroalimentación. Falta de información se refiere a situaciones cuando un orador en una actividad comunicativa posee información que el interlocutor no tiene; en este caso, los alumnos tienen la obligación de comunicarse utilizando la lengua meta con el fin de obtener la información que necesitan. La elección se refiere al hecho de que tenemos diferentes formas de decir lo mismo y los estudiantes deben tener la oportunidad de utilizar el idioma como lo deseen, siempre y cuando cumplan con el objetivo comunicativo; esto significa que los estudiantes nunca deben limitarse a la utilización de sólo una estructura para llevar a cabo una función particular. La retroalimentación es la respuesta que se obtiene al interactuar con alguien; esta es la característica más importante de las tres funciones, ya que es por este aspecto que el estudiante puede determinar si está logrando comunicar su mensaje.

\subsection{El enfoque humanístico afectivo}

Este tipo de enfoque se ha desarrollado como el más radical. En este programa, los estudiantes deciden lo que quieren aprender y hacer con la lengua meta. Este enfoque es la reacción a la falta general de las consideraciones afectivas en el Método Audiolingüístico (Celce-Murcia, 1991, p.7). Se hace hincapié en el respeto por el individuo. Uno de los aspectos más fuertes de este enfoque establece que si los profesores respetan a sus estudiantes y si los estudiantes aprenden a respetar a sus compañeros de clase, se creará un ambiente libre de estrés en el que tendrán la libertad de participar y dar sus propias opiniones. El respeto es uno de los pilares para reducir el filtro afectivo, para usar el término de Krashen, y al hacerlo los estudiantes aprenderán más. El ambiente de clase en este enfoque es aún más importante que los materiales o métodos y hasta cierto punto es cierto, pues un profesor puede tener un método de enseñanza excelente, un gran conocimiento de lo que está destinado a enseñar, pero si no puede crear un ambiente agradable para que los estudiantes puedan aprender, muchos de ellos no serán capaces de hacerlo adecuadamente.

"Trate a sus estudiantes como usted quiere ser tratado" es, talvez, el mejor lema para los profesores de lengua de acuerdo con Rogers en su enfoque de la Psicología Humanista (Brown, 1994, p.85). Según este autor, el sistema educativo niega la libertad y la dignidad de las personas, ya que se prescriben con las metas curriculares, objetivos y estrategias que tienen que aprender, incluso si esos objetivos no se ajustan a sus necesidades. Lo que se necesita, de acuerdo con Rogers, son facilitadores reales de aprendizaje, y esto se logra sólo cuando el 
facilitador establece relaciones interpersonales con el alumno. De esta manera, los profesores deben ser reales y dejar de pensar en la superioridad y la omnisciencia. En segundo lugar, los profesores necesitan ver a los estudiantes como individuos valiosos. Y en tercer lugar, deben comunicarse de manera abierta y enfática con sus alumnos y viceversa.

\section{Metodología de la investigación}

Los resultados de la presente investigación fueron obtenidos por medio de un cuestionario aplicado a un grupo de 47 profesores de enseñanza del inglés. Este cuestionario tiene un total de 11 preguntas (5 preguntas abiertas y 6 cerradas) que permiten extraer información tanto de índole cuantitativa como cualitativa. El objetivo principal de este instrumento es establecer una relación entre los enunciados teóricos más populares para la enseñanza de lenguas y su puesta en práctica. Además, será posible determinar el grado de aceptación que tienen estos enunciados dentro del grupo de docentes entrevistados.

\section{Análisis de resultados}

Entre los entrevistados, un 59.5\% tiene claro el concepto de aprendizaje, mientras que un $27.6 \%$ no; sin embargo, es interesante destacar que existe un grupo que alcanza el $12.9 \%$ que mezcla aspectos tanto de aprendizaje como de adquisición. Si bien es cierto, este aspecto se refiere al manejo de un concepto teórico, su importancia radicará en que los profesores puedan realizar la conexión para pasar de un proceso de aprendizaje a uno de adquisición. Al analizar las definiciones proporcionadas por los profesores, es posible determinar que en su mayoría estos tienen claros tres aspectos básicos sobre el concepto de aprendizaje, los cuales se establecen en el siguiente gráfico:

\section{Gráfico 1 \\ Aspectos básicos del concepto de aprendizaje}

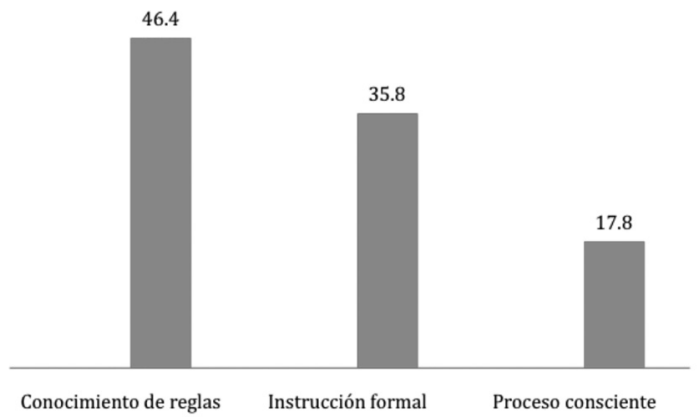


Por otro lado, al analizar el concepto que estos docentes proporcionan sobre adquisición, es posible determinar que un $44.6 \%$ de ellos tiene una idea clara de lo que implica este proceso, mientras que un $17 \%$ no puede proporcionar un concepto preciso. Es interesante que un $38.4 \%$ escribe un concepto que tiene elementos de adquisición como también de aprendizaje. Al analizar las respuestas proporcionadas por el $44.6 \%$ de los entrevistados, es posible destacar tres características básicas del proceso de adquisición que se establecen como esenciales y que se presentan en el siguiente gráfico:

\section{Gráfico 2 \\ Aspectos básicos del concepto de adquisición}

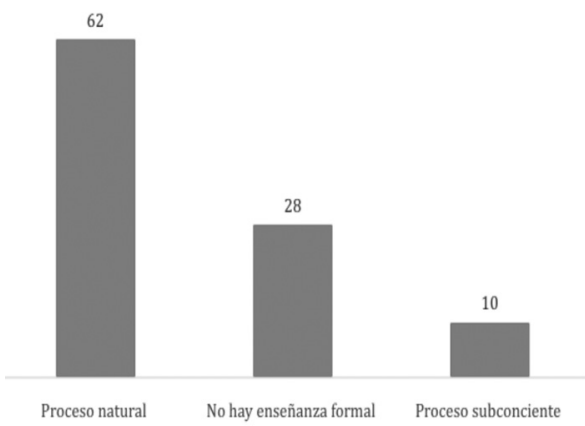

Quizás una de las secciones modulares de la encuesta se enfoca en la capacidad que tengan los entrevistados para planear lecciones que les permitan migrar de un proceso de aprendizaje a un proceso de adquisición. Es importante recalcar que la dinámica de clase está estrictamente relacionada con la acumulación de conocimiento lingüístico consciente, de ahí la importancia de que los profesores puedan proveer actividades donde el uso de la lengua meta se vuelva real y por lo tanto promueva la adquisición. El 42.50\% de los entrevistados asegura que el uso de diálogos en la clase permite migrar de un proceso de aprendizaje a uno de adquisición; un $29.80 \%$, por otro lado, indica que incrementar el conocimiento lingüístico nos permitirá cumplir con dicho cometido. La utilización de juegos es apoyada por un $6.5 \%$, mientras que propiciar el uso del lenguaje en forma realista fue favorecido por un $21.20 \%$ de los entrevistados. Si se realiza un análisis de la información supra indicada, se puede determinar que el incremento del conocimiento lingüístico no promocionará la adquisición del idioma por sí sola, ya que será necesario ligar dicho conocimiento con la puesta en práctica de una actividad natural y comunicativa.

Cuando los entrevistados tienen que identificar una serie de actividades con el tipo de proceso que se favorece, sea aprendizaje o adquisición, resulta claro que los profesores tienen un conocimiento apropiado de dichas actividades. Quizás se pueda concluir, en este aspecto, que los profesores poseen problemas únicamente para determinar si los ejercicios de escritura y los juegos propician la adquisición o el aprendizaje. Esta información queda reflejada en el siguiente cuadro: 


\section{Cuadro 1 \\ Actividades que favorecen el aprendizaje o la adquisición}

\begin{tabular}{lcc}
\hline \multicolumn{1}{c}{ Actividad } & Aprendizaje & Adquisición \\
\hline Diálogos & $19.15 \%$ & $80.85 \%$ \\
Ejercicios comunicativos y significativos & $11.00 \%$ & $89.00 \%$ \\
Ejercicios de escritura & $51.00 \%$ & $48.90 \%$ \\
Estrategias de comunicación & $23.50 \%$ & $76.50 \%$ \\
Enseñanza de la estructura del lenguaje & $74.50 \%$ & $25.50 \%$ \\
Juegos & $36.20 \%$ & $63.80 \%$ \\
Enseñanza de reglas lingüísticas & $87.20 \%$ & $12.80 \%$ \\
\hline
\end{tabular}

El proceso de monitoreo propuesto por Krashen ha sido ampliamente propiciado por este grupo de entrevistados, ya que el $80.80 \%$ de ellos asegura haber enseñado a sus estudiantes a monitorear su producción oral. Al preguntárseles sobre las estrategias utilizadas para motivar a sus estudiantes, un $42.50 \%$ de los profesores aseguran haberles indicado que los errores en sus presentaciones orales no serían penalizados si eran corregidos inmediatamente después de haber articulado un segmento erróneo. Un $32 \%$ indica que el monitoreo demuestra conocimiento de reglas lingüísticas, lo cual es percibido como positivo; un $21.20 \%$ indica que monitorear la producción oral mejora la capacidad la lingüística y un $4.39 \%$ indica que esta es una estrategia natural de aprendizaje.

A pesar de su importancia, la teoría del filtro afectivo propuesta por Krashen no es conocida por el $100 \%$ de los entrevistados, ya que un $59.60 \%$ indica conocer esta teoría y un $40.4 \%$ la desconoce. Del 59.60\% de los profesores que respondieron la encuesta, $28.60 \%$ considera medianamente importantes los alcances de la teoría del filtro afectivo, mientras que un $71.40 \%$ la considera importante. Esta información queda representada en el siguiente gráfico:

\section{Gráfico 3 \\ Importancia de la teoría del filtro afectivo}

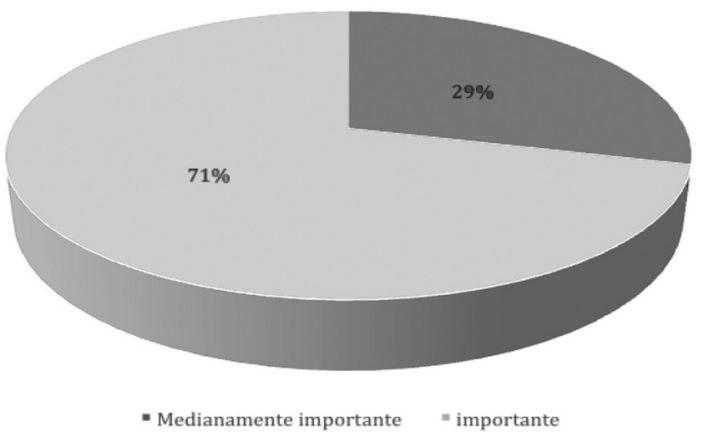


A los 28 profesores que manifestaron conocer la teoría del filtro afectivo se les solicitó indicar las técnicas que utilizan para disminuir el filtro afectivo de los estudiantes. El 64\% indica que crear un ambiente agradable propicia una baja en el filtro afectivo; un $26 \%$ manifiesta que propiciar el respeto entre los estudiantes ayuda significativamente en este proceso, y un $10 \%$ cree que tolerar los errores lingüísticos ayuda a los estudiantes. Es necesario indicar que esta tolerancia no ayuda a disminuir el filtro afectivo, pero sí la forma de corrección utilizada por el profesor.

Quizás uno de los aspectos de mayor importancia para asegurar el aprendizaje de los estudiantes es justamente desarrollar actividades que correspondan con el estilo de aprendizaje que poseen. La encuesta tiene una sección con varias actividades y los profesores debían indicar el tipo de estilo de aprendizaje que se vería beneficiado con estas actividades; sin embargo, llama la atención que algunos docentes tienen problemas en asociar el tipo de actividad con su correspondiente estilo de aprendizaje. Esta situación, evidentemente, causaría problemas a los estudiantes ya que el profesor estaría imposibilitado para desarrollar actividades que empaten con el estilo de aprendizaje de sus alumnos. En particular, los profesores tienen problemas para identificar actividades para estudiantes cuyos estilos de aprendizaje son impulsivo, reflexivo, campo dependiente y campo independiente. Esta información queda en evidencia en el siguiente gráfico:

\section{Gráfico 4 \\ Identificación de actividades según estilo de aprendizaje}

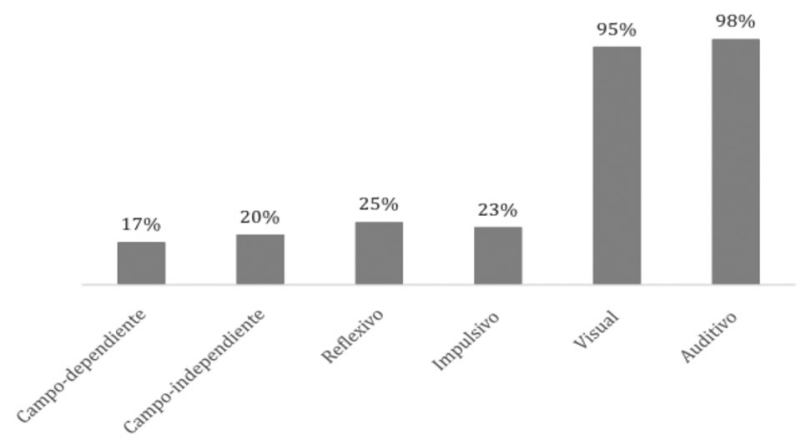

Cuando los profesores son cuestionados sobre la importancia de la cultura, muchos de ellos mencionan razones que concuerdan con las propuestas por Seelye (1993) en su libro Teaching Culture. Un grupo correspondiente al 42.50\% de los entrevistados indica que es importante enseñar cultura como una estrategia para preparar a los estudiantes a enfrentarse a la cultura meta de una forma satisfactoria; un $32.10 \%$ piensa que la cultura le permitirá a los estudiantes comprender que las variaciones sociales influyen en la forma en que hablamos y actuamos; un $14.80 \%$ cree que la enseñanza de la cultura les permite a los estudiantes comprender que las personas satisfacen sus necesidades de formas 
distintas y, por último, un $10.60 \%$ indica que la cultura ayuda a evitar generalizaciones negativas.

Basados en los resultados obtenidos en este estudio, es posible concluir que los profesores favorecen un enfoque ecléctico el cual combina diferentes estrategias de varios enfoques. Esto permite tener una amplia gama de actividades para poder desarrollar en el salón de clases y a la vez permite empatar con los diferentes estilos de aprendizaje de los estudiantes. En el siguiente gráfico es posible observar las tres actividades más utilizadas por estos profesores al igual que las tres menos empleadas.

\section{Gráfico 5 \\ Estrategias, actividades o técnicas utilizadas en la clase de lengua}

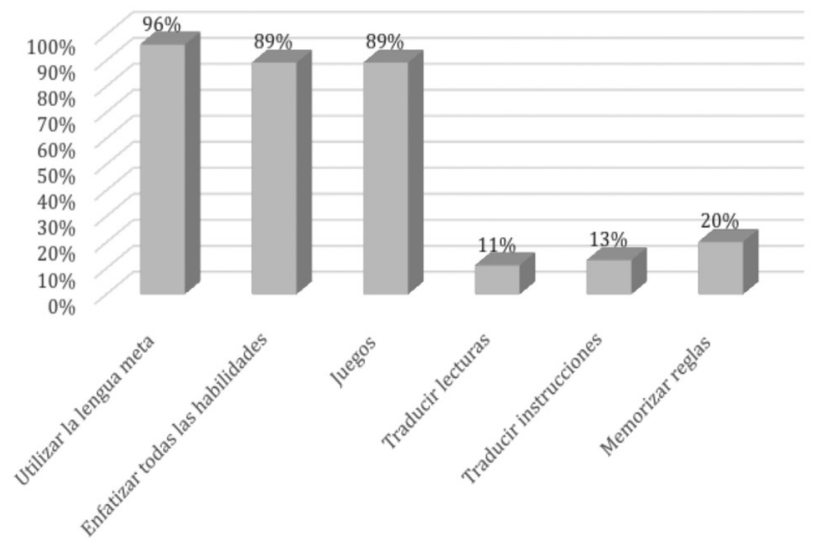

\section{Conclusiones}

Este artículo ha pretendido detallar algunos de los aspectos más relevantes sobre el proceso de enseñanza/aprendizaje de segundas lenguas y cómo los docentes llevan los enunciados teóricos a la práctica. En primera instancia, se puede determinar que el grupo de docentes analizado tiende a confundir los conceptos de adquisición y aprendizaje y, además, presenta problemas al identificar estrategias que les permitan migrar de un proceso de aprendizaje a un proceso de adquisición en sus lecciones. Esto quiere decir que se mantiene en el desarrollo de conocimiento lingüístico teórico sin permitirles a los estudiantes la puesta en práctica en contextos comunicativos significativos. Un grupo importante de los entrevistados propicia el uso de la autocorrección o monitoreo; sin embargo, cuando son cuestionados sobre las técnicas o estrategias utilizadas en clase para propiciar este proceso, se encuentra que los docentes no aplican estrategias certeras. Un porcentaje significativo reconoce la importancia de la teoría del filtro afectivo y propone medidas apropiadas para poder cumplir con este proceso; sin embargo, es importante indicar que una de las estrategias mencionadas es 
justamente la tolerancia en los errores lingüísticos que cometen los estudiantes, pero quizás los profesores se referían a utilizar una forma de corrección apropiada y no agresiva, más que a la tolerancia de errores, lo cual sería un verdadero problema en una clase de lengua extranjera. En cuanto a actividades basadas en los estilos de aprendizaje de los estudiantes, es necesario indicar que una mayoría presenta problemas para identificar actividades que propicien el desarrollo de un estilo de aprendizaje. Esto evidentemente trae problemas a algunos estudiantes cuyos estilos de aprendizaje no son compatibles con las actividades que se desarrollan en clase. En relación con el aspecto cultural, pareciera que los profesores tienen claros los objetivos de enseñar cultura en una clase de lengua extranjera e identifican como su principal razón la posibilidad de desarrollar en los estudiantes la capacidad de enfrentarse a la cultura meta de una manera satisfactoria. Por último y tomando en cuenta los enfoques para la enseñanza de lenguas, es claro que los profesores se basan en un enfoque ecléctico debido a la gran cantidad de actividades que utilizan en sus clases, las cuales son características de diferentes enfoques de aprendizaje. La ventaja de este enfoque es que permite al profesor una amplia gama de actividades para poder desarrollar en el salón de clases y, a la vez, le permite empatar con los diferentes estilos de aprendizaje de los estudiantes. Por último, se puede concluir que los profesores entrevistados hacen un manejo aceptable de los enunciados teóricos y sus implicaciones prácticas en el proceso de enseñanza y aprendizaje.

\section{Bibliografía}

Brown, H. D. (1994). Principles of Language Learning and Teaching. New Jersey: Prentice Hall Regents.

Brown, H. D. (1995). The Elements of Language Curriculum. Boston: Heinle y Heinle.

Celce-Murcia, M. (Ed.). (1991). Teaching English as a Second Language or Foreign Language. Boston: Heinle y Heinle.

Ellis, R. (1994). Understanding Second Language Learning. Oxford: Oxford University Press.

Gass, S. y Selinker, L. (1994). Second Language Acquisition: An Introductory Course. Hillsdale, New Jersey: Lawrence Erlbaum Associates, Publishers.

Hockett, C. (1967). A Course in Modern Linguistics. 12th ed. (1st ed. 1958). New York: The Macmillan Company, and Canada: Collier-Macmillan Ltd.

Johnson, K. y Morrow (1981). Communication in the Classroom. Essex: Longman.

Krashen, S. y Terrel, T. (1983). The Natural Approach. Pergamon Press.

Larsen-Freeman, D. (2000). Techniques and Principles in Language Teaching. Oxford: Oxford University Press.

Richards, J. y Rodgers, T. (1986). Approaches and Methods in Language Teaching. Cambridge: Cambridge University Press.

Seelye, H.N. (1993). Teaching Culture: Strategies for Intercultural Communication. Chicago: National Textbook Company. 


\section{Anexo}

\section{ENCUESTA PARA EL PROFESOR}

La siguiente encuesta tiene como objetivo obtener información sobre algunos de los enunciados teóricos más relevantes sobre el aprendizaje de segundas lenguas y cómo estos son finalmente aplicados en la realidad del aula.

La información que usted suministre será utilizada para realizar una investigación que podrá ser publicada.

1. ¿Qué es aprendizaje?

2. ¿Qué es adquisición?

3. ¿Cómo logra usted que sus estudiantes pasen de aprender el idioma a adquirirlo en sus clases?

4. Determine cuáles de las siguientes actividades favorecen el aprendizaje y cuáles la adquisición.

\begin{tabular}{|c|c|c|}
\hline & Aprendizaje & Adquisición \\
\hline \multicolumn{3}{|c|}{ Diálogos } \\
\hline \multicolumn{3}{|c|}{$\begin{array}{c}\text { Ejercicios comunicativos y } \\
\text { significativos }\end{array}$} \\
\hline \multicolumn{3}{|c|}{ Ejercicios de escritura } \\
\hline \multicolumn{3}{|c|}{ Estrategias de comunicación } \\
\hline \multicolumn{3}{|c|}{ Estructura del lenguaje } \\
\hline \multicolumn{3}{|c|}{ Juegos } \\
\hline Reglas & & \\
\hline
\end{tabular}

5. ¿Le ha enseñado usted a sus estudiantes a monitorear su producción oral?

( ) Sí (Pase a la pregunta 6)

( ) No (Pase a la pregunta 7)

6. ¿Qué tipo de estrategia utiliza usted para que sus estudiantes monitoreen su producción oral? 
7. ¿Conoce usted la teoría de filtro afectivo propuesta por Steven Krashen?

( ) Sí (Pase a la pregunta 8)

( ) No (Pase a la pregunta 10)

8. ¿Qué tan importante es para usted la aplicación de la teoría de filtro afectivo propuesta por Steven Krashen?

( ) Poco importante

( ) Medianamente importante

( ) Importante

9. ¿Cuáles técnicas utiliza usted para disminuir el filtro afectivo de los estudiantes?

10. Indique a cuál tipo de estilo de aprendizaje pertenecen las siguientes actividades.

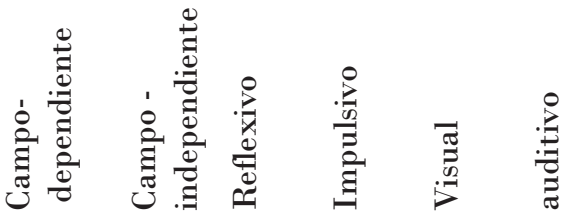

Escribir pequeños resúmenes de lo aprendido

Escuchar material de audio

Estudio de diagramas, esquemas, tablas

Instrucciones directas

Leer en voz alta

Pensar en preguntas o aplicaciones

Proyectos individuales

Reforzamiento de conductas correctas

Responder preguntas en forma oral

Tareas de trabajo cooperativo

Trabajo en grupo

Trabajo individual

11. ¿Por qué es importante la enseñanza de la cultura en una clase de lengua?

12. Indique con qué frecuencia aplica usted las siguientes estrategias o técnicas en sus clases de lengua. 
Con mucha

frecuencia
Con

frecuencia
Con poca

frecuencia

Utiliza la lengua meta en clase

Traduce lecturas

Traduce instrucciones

Enseña la gramática en forma deductiva

Memoriza reglas

Responde preguntas de comprensión de lectura

Escribe composiciones

Enseña cultura de forma inductiva

Se enfatizan todas las habilidades de la len-

gua

Se resta importancia a la pronunciación

La enseñanza se centra en funciones y estruc-

turas

Utiliza recursos lingüísticos auténticos

Utiliza juegos

Se promueve el respeto 